\title{
The inclusion theorem for multiple summing operators
}

\author{
by \\ DAVID PÉRez-García (Madrid)
}

\begin{abstract}
We prove that, for $1 \leq p \leq q<2$, each multiple $p$-summing multilinear operator between Banach spaces is also $q$-summing. We also give an improvement of this result for an image space of cotype 2 . As a consequence, we obtain a characterization of Hilbert-Schmidt multilinear operators similar to the linear one given by A. Pełczyński in 1967. We also give a multilinear generalization of Grothendieck's Theorem for GT spaces.
\end{abstract}

1. Introduction. Motivated by the importance of the theory of absolutely summing linear operators, there have been some attempts to generalize this concept and the related results and tools to the multilinear setting (see [1], [7], [21] or [23]). However, the results obtained were not completely satisfactory. Recently, M. C. Matos [11] and, independently, F. Bombal, I. Villanueva and the author [2], [17], [18] have defined and studied the class of multiple summing operators (although the origin of this class goes back to [23]). It is shown in these works that this class behaves better in many ways than the previous definitions of $p$-summing multilinear operators. In fact, this class has most of the main properties of its linear analogue. For example, we have

THEOREM 1.1. (i) Every multilinear operator from $\mathcal{L}_{\infty}$-spaces into an $\mathcal{L}_{1}$-space is multiple 2-summing [2].

(ii) Every multilinear operator from $\mathcal{L}_{1}$-spaces into a Hilbert space is multiple p-summing for $1 \leq p \leq 2$ [17].

(iii) The composition of linear 2-summing operators with a multiple 2summing multilinear operator is integral and even nuclear [16], [18].

(iv) $X_{1}, \ldots, X_{n}$ are $\mathcal{L}_{\infty}$-spaces if and only if, for every Banach space $Y$, the class of multiple 1-summing multilinear operators $T: X_{1} \times$ $\cdots \times X_{n} \rightarrow Y$ coincides with the class of integral operators [18].

2000 Mathematics Subject Classification: Primary 47H60; Secondary 46B25, 46C99.

Key words and phrases: Hilbert-Schmidt operators, $p$-summing operators, absolutely summing operators, multilinear operators, Grothendieck's theorem.

Partially supported by DGICYT grant BMF2001-1284. 
We also have close relations with the Hilbert-Schmidt multilinear operators. In fact, it is proved in [11] that

THEOREM 1.2. The class of Hilbert-Schmidt multilinear operators and the class of multiple 2-summing operators are identically isometric.

Theorem 1.3. For a multilinear operator $T: X_{1} \times \cdots \times X_{n} \rightarrow Y$, the following are equivalent:

(i) $T$ is Hilbert-Schmidt.

(ii) $T$ is multiple $p$-summing for every $p \in[2, \infty)$.

(iii) $T$ is multiple $p$-summing for some $p \in[2, \infty)$.

ThEOREM 1.4. Every Hilbert-Schmidt multilinear operator is multiple p-summing for every $1 \leq p<2$.

The class of Hilbert-Schmidt multilinear operators was introduced in [8], and studied and used in, for example, [3], [4], [9] or [21] (see Section 5 for more details).

However, as shown in [18], for every $q>2$ there exists a bilinear form that is multiple $p$-summing for every $1 \leq p \leq 2$ but not $q$-summing. Therefore, and this is one of the main defects of this class, we cannot expect an Inclusion Theorem similar to [6, Theorem 2.8]. The aim of this paper is to solve this problem partially by showing (see Theorem 3.4) that if $1 \leq p \leq q<2$, then every multiple $p$-summing operator is also multiple $q$-summing. In Theorem 3.10 we improve this result for the case of an image space of cotype 2 .

As a first application, we give in Theorem 4.2 a multilinear version of the classical characterization of Hilbert-Schmidt linear operators given by A. Pełczyński [15] (see also [19]). Thus, we complete the relations given in Theorems 1.2-1.4.

Moreover, we prove in Theorem 4.5 that, for GT spaces, every multilinear operator into a Hilbert space is 1-summing. Though this result can be obtained by using the approach of [2], this new proof gives us an optimal constant, improving the proofs in [2] and [17]. The Inclusion Theorem allows us to extend this result to $1 \leq p \leq 2$ and so we conclude that Theorem 1.1(ii) is also valid for GT spaces.

2. Notation and definitions. The notation and terminology used along the paper is standard in Banach space theory, as for instance in [5] or [6]. These books are also our main references for basic facts, definitions and unexplained notation. However, before going any further, we shall establish some terminology. All along this paper all the operators are supposed to be continuous. Given Banach spaces $X, Y, \mathcal{L}(X, Y)$ will denote the Banach space of linear (and continuous) operators between them and $X \otimes_{\varepsilon} Y$ will be their injective tensor product. $X^{*}$ will be the dual of $X$ and $B_{X}$ its unit ball. 
We will denote by $Y \stackrel{1}{\hookrightarrow} X$ an isometric inclusion and $X \stackrel{1}{=} Y$ will denote that $X$ and $Y$ are isometrically isomorphic. Given linear operators $u: X \rightarrow Y$ and $v: Z \rightarrow W, u \otimes v: X \otimes Z \rightarrow T \otimes W$ will be the linear operator given by $u \otimes v(x \otimes z)=u(x) \otimes v(z)$. As usual, $u^{*}$ will denote the adjoint operator of $u$; and $\mathbb{K}$ will denote the basic field, which can be both $\mathbb{R}$ and $\mathbb{C}$.

For a finite sequence $\left(x_{i}\right)_{i=1}^{m} \subset X$ and $1 \leq p<\infty$, we will write $\left\|\left(x_{i}\right)_{i=1}^{m}\right\|_{p}^{\omega}$ for

$$
\sup \left\{\left(\sum_{i=1}^{m}\left|x^{*}\left(x_{i}\right)\right|^{p}\right)^{1 / p}: x^{*} \in B_{X^{*}}\right\} .
$$

A linear operator $T: X \rightarrow Y$ is said to be $p$-summing if there exists a constant $K>0$ such that $\left(\sum_{i=1}^{m}\left\|T\left(x_{i}\right)\right\|^{p}\right)^{1 / p} \leq K\left\|\left(x_{i}\right)_{i=1}^{m}\right\|_{p}^{\omega}$ for each finite sequence $\left(x_{i}\right)_{i=1}^{m} \subset X$. We can extend this definition to the multilinear case:

Definition 2.1. Let $1 \leq p<\infty$. A multilinear operator $T: X_{1} \times \cdots \times$ $X_{n} \rightarrow Y$ is multiple p-summing if there exists a constant $K>0$ such that, for every choice of sequences $\left(x_{i_{j}}^{j}\right)_{i_{j}=1}^{m_{j}} \subset X_{j}$,

$$
\left(\sum_{i_{1}, \ldots, i_{n}=1}^{m_{1}, \ldots, m_{n}}\left\|T\left(x_{i_{1}}^{1}, \ldots, x_{i_{n}}^{n}\right)\right\|^{p}\right)^{1 / p} \leq K \prod_{j=1}^{n}\left\|\left(x_{i_{j}}^{j}\right)_{i_{j}=1}^{m_{j}}\right\|_{p}^{\omega} .
$$

In that case, we define the multiple p-summing norm of $T$ by

$$
\pi_{p}(T)=\min \{K: K \text { satisfies }(1)\} .
$$

The class $\Pi_{p}^{n}\left(X_{1}, \ldots, X_{n} ; Y\right)$ of multiple $p$-summing $n$-linear operators is a Banach space with the norm $\pi_{p}$.

Another class related to $p$-summing operators is the class of 2-dominated operators (see [5] or [6]). A linear operator $u: X \rightarrow Y$ is said to be 2dominated if there exist a Hilbert space $H$ and linear operators $v: X \rightarrow H$ and $w: H \rightarrow Y$ such that $u=w v$ and $v$ and $w^{*}$ are 2-summing. We define $\Delta_{2}(u)=\inf \left\{\pi_{2}(v) \pi_{2}\left(w^{*}\right)\right\}$, where the infimum is taken over all factorizations as above.

It is not difficult to see that $u: X \rightarrow Y$ is 2-dominated if and only if $u^{*}$ is. In this case $\Delta_{2}(u)=\Delta_{2}\left(u^{*}\right)$.

Grothendieck's Theorem says that there exists a universal constant $K>0$ such that every linear operator $u: \ell_{1} \rightarrow \ell_{2}$ is 1 -summing with $\pi_{1}(u) \leq K\|u\|$. We will denote by $K_{G}$ the least constant for which Grothendieck's Theorem is valid.

Following [22], we say that a Banach space $X$ is a $G T$ space, or that $X$ satisfies Grothendieck's Theorem, if there exists $K>0$ such that each linear operator $u: X \rightarrow \ell_{2}$ is 1 -summing and satisfies $\pi_{1}(u) \leq K\|u\|$. We denote the least such constant $K$ by GT $(X)$. According to Grothendieck's Theorem, $\mathcal{L}_{1}$ spaces are GT spaces, but there are several instances of GT 
spaces which are not $\mathcal{L}_{1}$ spaces, for example $L_{1} / H^{1}$ or the quotient of $L_{1}$ by a reflexive subspace (see [22]).

It is shown in [22] that $X$ is a GT space if and only if $X^{* *}$ is, and in that case, $\operatorname{GT}(X)=\mathrm{GT}\left(X^{* *}\right)$. Therefore, by [5, Proposition 20.18], every linear operator $u: X^{*} \rightarrow \ell_{1}$ is 2 -dominated and $\Delta_{2}(u) \leq \mathrm{GT}(X)\|u\|$. By the above remarks, we have

Proposition 2.2. If $X$ is a GT space, then every linear operator $u$ : $c_{0} \rightarrow X$ is 2-dominated and $\Delta_{2}(u) \leq \mathrm{GT}(X)\|u\|$.

For $q \geq 2$, a Banach space is said to have cotype $q$ if there exists a constant $K>0$ such that, for each choice of $\left(x_{i}\right)_{i=1}^{m} \subset X$, we have

$$
\left(\sum_{i=1}^{m}\left\|x_{i}\right\|^{q}\right)^{1 / q} \leq K\left(\int_{0}^{1}\left\|\sum_{i=1}^{m} r_{i}(t) x_{i}\right\|^{2} d t\right)^{1 / 2},
$$

where $\left(r_{i}\right)_{i=1}^{\infty}$ is the sequence of Rademacher functions. The least such constant $K$ will be denoted by $C_{q}(X)$.

Finally, we recall Khinchin's and Kahane's inequalities.

Theorem 2.3 (Khinchin's Inequality). For each $0<p<\infty$, there are positive constants $A_{p}, B_{p}$ such that, for each sequence $\left(a_{i}\right)_{i=1}^{\infty} \in \ell_{2}$, we have

$$
A_{p}\left(\sum_{i=1}^{\infty}\left|a_{i}\right|^{2}\right)^{1 / 2} \leq\left(\int_{0}^{1}\left|\sum_{i=1}^{\infty} a_{i} r_{i}(t)\right|^{p} d t\right)^{1 / p} \leq B_{p}\left(\sum_{i=1}^{\infty}\left|a_{i}\right|^{2}\right)^{1 / 2} .
$$

Theorem 2.4 (Kahane's Inequality). If $0<p, q<\infty$, there exists a constant $K_{p, q}>0$ such that

$$
\left(\int_{0}^{1}\left\|\sum_{i=1}^{m} r_{i}(t) x_{i}\right\|^{q} d t\right)^{1 / q} \leq K_{p, q}\left(\int_{0}^{1}\left\|\sum_{i=1}^{m} r_{i}(t) x_{i}\right\|^{p} d t\right)^{1 / p}
$$

for each Banach space $X$ and each choice of elements $x_{1}, \ldots, x_{m} \in X$.

More notation and terminology will be introduced when needed.

3. Main results. To give the proof of the main result of this paper, we will need an integral formulation of the definition of multiple summing operators, in the spirit of [22, Proposition 1.2].

Proposition 3.1. Let $T \in \Pi_{p}^{n}\left(X_{1}, \ldots, X_{n} ; Y\right)$ and let $\left(\Omega_{j}, \Sigma_{j}, \mu_{j}\right)$ be measure spaces for each $1 \leq j \leq n$. Then

$$
\begin{gathered}
\left(\int_{\Omega_{1}} \cdots \int_{\Omega_{n}}\left\|T\left(f_{1}\left(\omega_{1}\right), \ldots, f_{n}\left(\omega_{n}\right)\right)\right\|^{p} d \mu_{n}\left(\omega_{n}\right) \cdots d \mu_{1}\left(\omega_{1}\right)\right)^{1 / p} \\
\leq \pi_{p}(T) \prod_{j=1}^{n} \sup _{x^{*} \in B_{X^{*}}}\left(\int_{\Omega_{j}}\left|\left\langle x^{*}, f_{j}\left(\omega_{j}\right)\right\rangle\right|^{p} d \mu_{j}\left(\omega_{j}\right)\right)^{1 / p}
\end{gathered}
$$

for every $f_{j} \in L_{p}\left(\mu_{j}, X_{j}\right)$. 
Proof. It is enough to prove the result for simple functions of the form $f_{j}=\sum_{i_{j}=1}^{m_{j}} x_{i_{j}}^{j} \chi_{A_{i_{j}}^{j}}$, where, for each $j$, the sets $A_{i_{j}}^{j} \subset \Omega_{j}$ are measurable and disjoint and $x_{i_{j}}^{j} \in X_{j}$. Then (2) reduces to

$$
\begin{aligned}
& \left(\sum_{i_{1}, \ldots, i_{n}=1}^{m_{1}, \ldots, m_{n}}\left\|T\left(\mu_{1}\left(A_{i_{1}}^{1}\right)^{1 / p} x_{i_{1}}^{1}, \ldots, \mu_{n}\left(A_{i_{n}}^{n}\right)^{1 / p} x_{i_{n}}^{n}\right)\right\|^{p}\right)^{1 / p} \\
& \leq \pi_{p}(T) \prod_{j=1}^{n} \sup _{x^{*} \in B_{X^{*}}}\left(\sum_{i_{j}=1}^{m_{j}}\left|\left\langle x^{*}, \mu_{j}\left(A_{i_{j}}^{j}\right)^{1 / p} x_{i_{j}}^{j}\right\rangle\right|^{p}\right)^{1 / p},
\end{aligned}
$$

which is true by Definition 2.1.

Our main tool will be probability in Banach spaces. For this, all we will need is covered by [20, Chapter 21].

The first result we will need is the existence of $q$-stable random variables:

Proposition 3.2. For $1 \leq p<q \leq 2$, there exist a positive constant $c_{p, q}$, a measure space $(\Omega, \Sigma, \mu)$ and a sequence $\left(\theta_{i}\right)_{i=1}^{\infty}$ of measurable scalar-valued functions such that

$$
\left\|\sum_{i=1}^{m} \alpha_{i} \theta_{i}\right\|_{L_{p}(\mu)}=c_{p, q}\left(\sum_{i=1}^{m}\left|\alpha_{i}\right|^{q}\right)^{1 / q}
$$

for each $\left(\alpha_{i}\right)_{i=1}^{m} \subset \mathbb{K}$.

Moreover, we will need the following fact, due to Maurey [12], which essentially says that every Banach space has cotype $(q, p)$ for $1 \leq p<q<2$ :

Proposition 3.3. If $1 \leq p<q<2$, there exists a constant $\widetilde{K}_{p, q}>0$, which only depends on $p$ and $q$, such that if $Z$ is a Banach space and $\left(z_{i}\right)_{i=1}^{m} \subset Z$, then

$$
\left(\sum_{i=1}^{m}\left\|z_{i}\right\|^{q}\right)^{1 / q} \leq \widetilde{K}_{p, q}\left(\int\left\|\sum_{\Omega}^{m} \theta_{i}(t) z_{i}\right\|^{p} d \mu(t)\right)^{1 / p} .
$$

We set $K_{p, q}=c_{p, q} \widetilde{K}_{p, q}$.

Theorem 3.4. Let $1 \leq p<q<2$. If $X_{1}, \ldots, X_{n}, Y$ are Banach spaces and $T \in \Pi_{p}^{n}\left(X_{1}, \ldots, X_{n} ; Y\right)$, then $T \in \Pi_{q}^{n}\left(X_{1}, \ldots, X_{n} ; Y\right)$ and $\pi_{q}(T) \leq$ $K_{p, q}^{n} \pi_{p}(T)$.

Proof. Take $T \in \Pi_{p}^{n}\left(X_{1}, \ldots, X_{n} ; Y\right)$ and $\left(x_{i_{j}}^{j}\right)_{i_{j}=1}^{m_{j}} \subset X_{j}$. We are going to show that

$$
\left(\sum_{i_{1}, \ldots, i_{n}=1}^{m_{1}, \ldots, m_{n}}\left\|T\left(x_{i_{1}}^{1}, \ldots, x_{i_{n}}^{n}\right)\right\|^{q}\right)^{1 / q} \leq K_{p, q}^{n} \pi_{p}(T) \prod_{j=1}^{n}\left\|\left(x_{i_{j}}^{j}\right)_{i_{j}=1}^{m_{j}}\right\|_{q}^{\omega} .
$$


By (4), it is not difficult to see by induction that

$$
\begin{aligned}
& \left(\sum_{i_{1}, \ldots, i_{n}=1}^{m_{1}, \ldots, m_{n}}\left\|T\left(x_{i_{1}}^{1}, \ldots, x_{i_{n}}^{n}\right)\right\|^{q}\right)^{1 / q} \\
& \leq \widetilde{K}_{p, q}^{n}\left(\int_{\Omega} \cdots \int_{\Omega}\left\|T\left(\sum_{i_{1}=1}^{m_{1}} \theta_{i_{1}}\left(\omega_{1}\right) x_{i_{1}}^{1}, \ldots, \sum_{i_{n}=1}^{m_{n}} \theta_{i_{n}}\left(\omega_{n}\right) x_{i_{n}}^{n}\right)\right\|^{p}\right. \\
& \left.d \mu\left(\omega_{n}\right) \cdots d \mu\left(\omega_{1}\right)\right)^{1 / p}
\end{aligned}
$$

Then Proposition 3.1 tells us that

$$
\begin{aligned}
& \left(\sum_{i_{1}, \ldots, i_{n}=1}^{m_{1}, \ldots, m_{n}}\left\|T\left(x_{i_{1}}^{1}, \ldots, x_{i_{n}}^{n}\right)\right\|^{q}\right)^{1 / q} \\
& \quad \leq \widetilde{K}_{p, q}^{n} \pi_{p}(T) \prod_{j=1}^{n} \sup _{x^{*} \in B_{X^{*}}}\left(\int\left|\sum_{\Omega}^{m_{j}} \theta_{i_{j}=1}\left(\omega_{j}\right)\left\langle x^{*}, x_{i_{j}}^{j}\right\rangle\right|^{p} d \mu\left(\omega_{j}\right)\right)^{1 / p}
\end{aligned}
$$

and using (3) we obtain (5).

REMARK 3.5. It is important to note that we cannot ensure that the constant $K_{p, q}$ in Theorem 3.4 is bounded as $q$ increases to 2 .

We can improve the constant in Theorem 3.4 using a tricky induction argument. This new result gives the constant 1 in the case $n=1$.

Theorem 3.6. Let $1 \leq p<q<2$. If $X_{1}, \ldots, X_{n}, Y$ are Banach spaces and $T \in \Pi_{p}^{n}\left(X_{1}, \ldots, X_{n} ; Y\right)$, then $T \in \Pi_{q}^{n}\left(X_{1}, \ldots, X_{n} ; Y\right)$ and $\pi_{q}(T) \leq$ $K_{p, q}^{n-1} \pi_{p}(T)$.

The induction step will use the following lemma, which is trivial from the definitions.

Lemma 3.7. Let $T: X_{1} \times \cdots \times X_{n} \rightarrow Y$ be a multilinear operator and let $1 \leq p<\infty$. The following are equivalent:

(i) $T$ is multiple p-summing.

(ii) There exists a constant $K>0$ such that for every finite sequence $\left(x_{i}^{1}\right)_{i=1}^{m} \subset X_{1}$ with $\left\|\left(x_{i}^{1}\right)_{i=1}^{m}\right\|_{p}^{\omega} \leq 1$ the associated $(n-1)$-linear operator

$$
T_{\left(x_{i}^{1}\right)}: X_{2} \times \cdots \times X_{n} \rightarrow \ell_{q}^{m}(Y)
$$

given by

$$
T_{\left(x_{i}^{1}\right)}\left(x^{2}, \ldots, x^{n}\right)=\left(T\left(x_{i}^{1}, x^{2}, \ldots, x^{n}\right)\right)_{i=1}^{m}
$$

is multiple p-summing and satisfies

$$
\pi_{p}\left(T_{\left(x_{i}^{1}\right)}\right) \leq K .
$$

In that case, $\pi_{p}(T)=\min \{K: K$ satisfies $(6)\}$. 
Proof of Theorem 3.6. We reason by induction. The case $n=1$ is $[6$, Theorem 2.8]. To deduce the $n$-case from the $(n-1)$-case, we will use Lemma 3.7. So consider $\left(x_{i}^{1}\right)_{i=1}^{m} \subset X_{1}$ such that $\left\|\left(x_{i}^{1}\right)_{i=1}^{m}\right\|_{q}^{w} \leq 1$. We want to show that

$$
\pi_{q}\left(T_{\left(x_{i}^{1}\right)}\right) \leq K_{p, q}^{n-1} \pi_{p}(T) \text {. }
$$

Set $h_{i}=\theta_{i} / c_{p, q}, E=\operatorname{span}\left\{h_{1}, \ldots, h_{m}\right\} \subset L_{p}(\mu)$ and let $h: \ell_{q}^{m} \stackrel{1}{\hookrightarrow} E$ be the isometric inclusion given by $e_{i} \mapsto h_{i}$. Then

$$
\|h\|=\left\|h^{-1}\right\|=1 \text {. }
$$

Let $\varepsilon>0$. Choose simple functions $S_{i} \in L_{p}(\mu)$ such that $\left\|S_{i}-h_{i}\right\|_{L_{p}(\mu)} \leq$ $\varepsilon / m$. Clearly, there exist $A_{1}, \ldots, A_{N} \subset \Omega$, measurable and disjoint, such that

$$
S_{i}=\sum_{j=1}^{N} \frac{\alpha_{i}^{j}}{\mu\left(A_{j}\right)^{1 / p}} \chi_{A_{j}} \quad \text { for } i=1, \ldots, m .
$$

We define $u: E \rightarrow \ell_{p}^{N}$ by $u\left(h_{i}\right)=\left(\alpha_{i}^{j}\right)_{j=1}^{N}$ and $v: \ell_{p}^{N} \stackrel{1}{\hookrightarrow} L_{p}(\mu)$ by $v\left(e_{j}\right)=$ $\chi_{A_{j}} / \mu\left(A_{j}\right)^{1 / p}$. It is clear that $v$ is an isometric inclusion. Moreover,

$$
\|u\| \leq 1+\varepsilon
$$

because

$$
\begin{aligned}
\left\|u\left(\sum_{i=1}^{m} \beta_{i} h_{i}\right)\right\|_{\ell_{p}^{N}} & =\left\|v \circ u\left(\sum_{i=1}^{m} \beta_{i} h_{i}\right)\right\|_{L_{p}(\mu)}=\left\|\sum_{i=1}^{m} \beta_{i} S_{i}\right\|_{L_{p}(\mu)} \\
& \leq\left\|\sum_{i=1}^{m} \beta_{i} h_{i}\right\|_{L_{p}(\mu)}+\left\|\sum_{i=1}^{m} \beta_{i}\left(S_{i}-h_{i}\right)\right\|_{L_{p}(\mu)} \\
& \leq\left\|\sum_{i=1}^{m} \beta_{i} h_{i}\right\|_{L_{p}(\mu)}+\left(\sum_{i=1}^{m}\left|\beta_{i}\right|^{q}\right)^{1 / q} \sum_{i=1}^{m}\left\|S_{i}-h_{i}\right\|_{L_{p}(\mu)} \\
& \leq(1+\varepsilon)\left\|\sum_{i=1}^{m} \beta_{i} h_{i}\right\|_{L_{p}(\mu)} \text { by (8). }
\end{aligned}
$$

Moreover, for $y=\sum_{i=1}^{n} h_{i} \otimes y_{i} \in L_{p}(\mu, Y)$, by (8) and [5] we have

$$
\max _{i}\left\|y_{i}\right\|_{Y} \leq\left\|\sum_{i=1}^{n} e_{i} \otimes y_{i}\right\|_{\ell_{q}^{m} \otimes_{\varepsilon} Y} \leq\left\|\sum_{i=1}^{n} h_{i} \otimes y_{i}\right\|_{E \otimes_{\varepsilon} Y} \leq\|y\|_{L_{p}(\mu, Y)} .
$$

Therefore,

$$
\begin{aligned}
(1-\varepsilon)\|y\|_{L_{p}(\mu, Y)} & \leq\|y\|_{L_{p}(\mu, Y)}-\sum_{i=1}^{m}\left\|y_{i}\right\|_{Y}\left\|h_{i}-S_{i}\right\|_{L_{p}(\mu)} \\
& \leq\left\|\sum_{i=1}^{m} S_{i} \otimes y_{i}\right\|_{L_{p}(\mu, Y)}=\|(v \circ u) \otimes \operatorname{id}(y)\|_{L_{p}(\mu, Y)} .
\end{aligned}
$$


Finally, using (4) we see that, for $y=\sum_{i=1}^{m} e_{i} \otimes y_{i} \subset \ell_{q}^{m}(Y)$,

$$
\|h \otimes \operatorname{id}(y)\|_{L_{p}(\mu, Y)} \geq \frac{1}{c_{p, q} \widetilde{K}_{p, q}}\|y\|_{\ell_{q}^{m}(Y)} .
$$

We can now prove that $\pi_{q}\left(T_{\left(x_{i}^{1}\right)}\right) \leq K_{p, q}^{n-1} \pi_{p}(T)$.

By (8) and (9) the linear operator $w=u \circ h: \ell_{q}^{m} \rightarrow \ell_{p}^{N}$ satisfies $\|w\| \leq$ $1+\varepsilon$. Denote by $l: \ell_{q^{*}}^{m} \rightarrow X_{1}$ the linear operator defined by $l\left(e_{i}\right)=x_{i}^{1}$. By [6, Proposition 2.2], $\|l\|=\left\|\left(x_{i}^{1}\right)_{i=1}^{m}\right\|_{q}^{w} \leq 1$ and so $\left\|l \circ w^{*}\right\| \leq 1+\varepsilon$.

Now, $l \circ w^{*}: \ell_{p^{*}}^{N} \rightarrow X_{1}$ and so if we set $z_{j}^{1}=l \circ w^{*}\left(e_{j}\right)$, then $\left\|\left(z_{j}^{1}\right)_{j=1}^{N}\right\|_{p}^{\omega} \leq$ $1+\varepsilon$, again by [6, Proposition 2.2]. But $z_{j}^{1}=\sum_{i=1}^{m} \alpha_{i}^{j} x_{i}^{1}$ for each $1 \leq j \leq N$ and $(w \otimes \mathrm{id}) \circ T_{\left(x_{i}^{1}\right)}: X_{2} \times \cdots \times X_{n} \rightarrow \ell_{p}^{N}(Y)$ is given by

$$
(w \otimes \mathrm{id}) \circ T_{\left(x_{i}^{1}\right)}\left(x^{2}, \ldots, x^{n}\right)=\left(T\left(\sum_{i=1}^{m} \alpha_{i}^{j} x_{i}^{1}, x^{2}, \ldots, x^{n}\right)\right)_{j=1}^{N} .
$$

Consequently, $(w \otimes \mathrm{id}) \circ T_{\left(x_{i}^{1}\right)}=T_{\left(z_{j}^{1}\right)}: X_{2} \times \cdots \times X_{n} \rightarrow \ell_{p}^{N}(Y)$, and we can deduce from Lemma 3.7 that

$$
\pi_{p}\left((w \otimes \mathrm{id}) \circ T_{\left(x_{i}^{1}\right)}\right) \leq(1+\varepsilon) \pi_{p}(T) .
$$

We now consider $\left(x_{i_{2}}^{2}\right)_{i_{2}=1}^{m_{2}} \subset X_{2}, \ldots,\left(x_{i_{n}}^{n}\right)_{i_{n}=1}^{m_{n}} \subset X_{n}$ such that

$$
\left\|\left(x_{i_{j}}^{j}\right)_{i_{j}=1}^{m_{j}}\right\|_{p}^{\omega} \leq 1 \quad \text { for each } 2 \leq j \leq n .
$$

We have

$$
\begin{aligned}
& \left(\sum_{i_{2}, \ldots, i_{n}=1}^{m_{2}, \ldots, m_{n}}\left\|T_{\left(x_{i}^{1}\right)}\left(x_{i_{2}}^{2}, \ldots, x_{i_{n}}^{n}\right)\right\|_{\ell_{q}^{m}(Y)}^{p}\right)^{1 / p} \\
& \quad \leq K_{p, q}\left(\sum_{i_{2}, \ldots, i_{n}=1}^{m_{2}, \ldots, m_{n}}\left\|(h \otimes \mathrm{id}) \circ T_{\left(x_{i}^{1}\right)}\left(x_{i_{2}}^{2}, \ldots, x_{i_{n}}^{n}\right)\right\|_{L_{p}(\mu, Y)}^{p}\right)^{1 / p} \quad \text { by }(11) \\
& \quad \leq \frac{K_{p, q}}{1-\varepsilon}\left(\sum_{i_{2}, \ldots, i_{n}=1}^{m_{2}, \ldots, m_{n}}\left\|(v u \otimes \mathrm{id}) \circ(h \otimes \mathrm{id}) \circ T_{\left(x_{i}^{1}\right)}\left(x_{i_{2}}^{2}, \ldots, x_{i_{n}}^{n}\right)\right\|_{L_{p}(\mu, Y)}^{p}\right)^{1 / p} \\
& \quad=\frac{K_{p, q}}{1-\varepsilon}\left(\sum_{i_{2}, \ldots, i_{n}=1}^{m_{2}, \ldots, m_{n}}\left\|(w \otimes \mathrm{id}) \circ T_{\left(x_{i}^{1}\right)}\left(x_{i_{2}}^{2}, \ldots, x_{i_{n}}^{n}\right)\right\|_{\ell_{p}^{N}(Y)}^{p}\right)^{1 / p} \quad \text { as } v \otimes \mathrm{id} \text { is an isometry } \\
& \quad \leq \frac{K_{p, q}}{1-\varepsilon}(1+\varepsilon) \pi_{p}(T) \quad \text { by }(12) .
\end{aligned}
$$


Therefore, as $\varepsilon$ is arbitrary, we have $\pi_{p}\left(T_{\left(x_{i}^{1}\right)}\right) \leq K_{p, q} \pi_{p}(T)$. Now the induction hypothesis tells us that $\pi_{q}\left(T_{\left(x_{i}^{1}\right)}\right) \leq K_{p, q}^{n-2} \pi_{p}\left(T_{\left(x_{i}^{1}\right)}\right)$ and we obtain the estimate (7).

We can improve Theorem 3.4 if the target space has cotype 2. First of all, we can cover the case $q=2$. What is even more important, we obtain uniform bounds for the inclusions (we have seen in Remark 3.5 that we do not have this in general).

We will need the following multiple cotype inequality.

Proposition 3.8. If $Y$ has cotype $2, p \leq 2$ and $\left(y_{i_{1}, \ldots, i_{n}}\right)_{i_{n}, \ldots, i_{n}=1}^{m_{1}, \ldots, m_{n}} \subset Y$, then

$$
\begin{aligned}
& \left(\sum_{i_{1}, \ldots, i_{n}=1}^{m_{1}, \ldots, m_{n}}\left\|y_{i_{1}, \ldots, i_{n}}\right\|^{2}\right)^{1 / 2} \\
& \leq\left(K_{p, 2} C_{2}(Y)\right)^{n}\left(\int_{0}^{1} \cdots \int_{0}^{1}\left\|\sum_{i_{1}, \ldots, i_{n}=1}^{m_{1}, \ldots, m_{n}} r_{i_{1}}\left(t_{1}\right) \cdots r_{i_{n}}\left(t_{n}\right) y_{i_{1}, \ldots, i_{n}}\right\|^{p} d t_{1} \cdots d t_{n}\right)^{1 / p},
\end{aligned}
$$

where $K_{p, 2}$ is the constant in Kahane's inequality 2.4 .

Proof. We argue by induction. The case $n=1$ is just the definition of cotype and Kahane's inequality. Let us prove case $n$ assuming case $n-1$. Again by the definition of cotype and Kahane's inequality we get

$$
\begin{aligned}
& \left(K_{p, 2} C_{2}(Y)\right)^{p} \int_{0}^{1} \cdots \int_{0}^{1}\left\|\sum_{i_{1}, \ldots, i_{n}=1}^{m_{1}, \ldots, m_{n}} r_{i_{1}}\left(t_{1}\right) \cdots r_{i_{n}}\left(t_{n}\right) y_{i_{1}, \ldots, i_{n}}\right\|^{p} d t_{1} \cdots d t_{n} \\
& \quad \geq \int_{0}^{1} \cdots \int_{0}^{1}\left(\sum_{i_{n}=1}^{m_{n}}\left\|\sum_{i_{1}, \ldots, i_{n-1}=1}^{m_{1}, \ldots, m_{n-1}} r_{i_{1}}\left(t_{1}\right) \cdots r_{i_{n-1}}\left(t_{n-1}\right) y_{i_{1}, \ldots, i_{n}}\right\|^{2}\right)^{p / 2} d t_{1} \cdots d t_{n-1}
\end{aligned}
$$

and using Minkowski's inequality for integrals, we obtain

$$
\begin{gathered}
\int_{0}^{1} \cdots \int_{0}^{1}\left(\sum_{i_{n}=1}^{m_{n}}\left\|\sum_{i_{1}, \ldots, i_{n-1}=1}^{m_{1}, \ldots, m_{n-1}} r_{i_{1}}\left(t_{1}\right) \cdots r_{i_{n-1}}\left(t_{n-1}\right) y_{i_{1}, \ldots, i_{n}}\right\|^{p \frac{2}{p}}\right)^{p / 2} d t_{1} \cdots d t_{n-1} \\
\geq\left(\sum _ { i _ { n } = 1 } ^ { m _ { n } } \left(\int_{0}^{1} \cdots \int_{0}^{1}\left\|\sum_{i_{1}, \ldots, i_{n-1}=1}^{m_{1}, \ldots, m_{n-1}} r_{i_{1}}\left(t_{1}\right) \cdots r_{i_{n-1}}\left(t_{n-1}\right) y_{i_{1}, \ldots, i_{n}}\right\|^{p}\right.\right. \\
\left.\left.d t_{1} \cdots d t_{n-1}\right)^{2 / p}\right)^{p / 2} .
\end{gathered}
$$

The induction hypothesis yields the result.

Using Minkowski's inequality exactly as in the proof of Proposition 3.8 we can obtain the following multiple version of Proposition 3.2. 
Proposition 3.9. If $\left(\alpha_{i_{1}, \ldots, i_{n}}\right)_{i_{1}, \ldots, i_{n}=1}^{m_{1}, \ldots, m_{n}} \subset \mathbb{K}$, then

$$
\begin{array}{r}
\left(\int_{\Omega} \cdots \int_{\Omega}\left|\sum_{i_{1}, \ldots, i_{n}=1}^{m_{1}, \ldots, m_{n}} \alpha_{i_{1}, \ldots, i_{n}} \theta_{i_{1}}\left(\omega_{1}\right) \cdots \theta_{i_{n}}\left(\omega_{n}\right)\right|^{p} d \mu\left(\omega_{1}\right) \cdots d \mu\left(\omega_{n}\right)\right)^{1 / p} \\
\geq c_{p, q}^{n}\left(\sum_{i_{1}, \ldots, i_{n}=1}^{m_{1}, \ldots, m_{n}}\left|\alpha_{i_{1}, \ldots, i_{n}}\right|^{q}\right)^{1 / q} .
\end{array}
$$

Clearly, we also have (3) and (13) for $\left(\varepsilon_{i} \theta_{i}\right)_{i=1}^{\infty}$, independently of the choice of signs $\left(\varepsilon_{i}\right)_{i=1}^{\infty} \in\{-1,1\}^{\mathbb{N}}$.

We can now prove our improvement of Theorem 3.4.

Theorem 3.10. If $1 \leq p<q \leq 2$, the space $Y$ has cotype 2 and $T \in$ $\Pi_{p}^{n}\left(X_{1}, \ldots, X_{n} ; Y\right)$, then $T \in \Pi_{q}^{n}\left(X_{1}, \ldots, X_{n} ; Y\right)$ and

$$
\pi_{q}(T) \leq\left(K_{p, 2} C_{2}(Y)\right)^{n} \pi_{p}(T),
$$

where $K_{p, 2}$ is the constant in Kahane's inequality 2.4 .

Proof. Using Proposition 3.2 and reasoning as in the proof of Theorem 3.4 , we obtain

$$
\begin{aligned}
& \left(\int_{0}^{1} \cdots \int_{0} \int_{\Omega}^{1} \cdots \int_{\Omega} \| \sum_{i_{1}, \ldots, i_{n}=1}^{m_{1}, \ldots, m_{n}} r_{i_{1}}\left(t_{1}\right) \cdots r_{i_{n}}\left(t_{n}\right) \theta_{i_{1}}\left(\omega_{1}\right) \cdots\right. \\
& \left.\cdots \theta_{i_{n}}\left(\omega_{n}\right) T\left(x_{i_{1}}^{1}, \ldots, x_{i_{n}}^{n}\right) \|^{p} d \mu\left(\omega_{1}\right) \cdots d \mu\left(\omega_{n}\right) d t_{1} \cdots d t_{n}\right)^{1 / p} \\
& \leq \pi_{p}(T) c_{p, q}^{n} \prod_{j=1}^{n}\left\|\left(x_{i_{j}}^{j}\right)_{i_{j}=1}^{m_{j}}\right\|_{q}^{\omega} .
\end{aligned}
$$

Using now Proposition 3.8 we find that the left side of (14) is greater than or equal to

$$
\begin{aligned}
& \frac{1}{\left(\left(K_{p, 2} C_{2}(Y)\right)^{n}\right.}\left(\int _ { \Omega } \cdots \int _ { \Omega } \left(\sum_{i_{1}, \ldots, i_{n}=1}^{m_{1}, \ldots, m_{n}} \mid \theta_{i_{1}}\left(\omega_{1}\right) \cdots\right.\right. \\
& \left.\left.\left.\cdots \theta_{i_{n}}\left(\omega_{n}\right)\left\|T\left(x_{i_{1}}^{1}, \ldots, x_{i_{n}}^{n}\right)\right\|\right|^{2}\right)^{p / 2} d \mu\left(\omega_{1}\right) \cdots d \mu\left(\omega_{n}\right)\right)^{1 / p}
\end{aligned}
$$

Now, using Khinchin's inequality 2.3, together with the fact that $B_{p}=1$, we see that (15) is again greater than or equal to

$$
\begin{aligned}
& \frac{1}{\left(K_{p, 2} C_{2}(Y)\right)^{n}}\left(\left.\int_{\Omega} \cdots \iint_{\Omega}^{1} \cdots \int_{0}^{1}\right|_{0} ^{1} \sum_{i_{1}, \ldots, i_{n}=1}^{m_{1}, \ldots, m_{n}} r_{i_{1}}\left(t_{1}\right) \cdots r_{i_{n}}\left(t_{n}\right) \theta_{i_{1}}\left(\omega_{1}\right) \cdots\right. \\
& \left.\left.\cdots \theta_{i_{n}}\left(\omega_{n}\right)\left\|T\left(x_{i_{1}}^{1}, \ldots, x_{i_{n}}^{n}\right)\right\|\right|^{p} d t_{1} \cdots d t_{n} d \mu\left(\omega_{1}\right) \cdots d \mu\left(\omega_{n}\right)\right)^{1 / p}
\end{aligned}
$$


and Proposition 3.9 shows that (16) is greater than or equal to

$$
\frac{1}{\left(K_{p, 2} C_{2}(Y)\right)^{n}} c_{p, q}^{n}\left(\sum_{i_{1}, \ldots, i_{n}=1}^{m_{1}, \ldots, m_{n}}\left\|T\left(x_{i_{1}}^{1}, \ldots, x_{i_{n}}^{n}\right)\right\|^{q}\right)^{1 / q}
$$

which finishes the proof.

REMARK 3.11. By [10], we can take $K_{p, 2}=\sqrt{2}$.

4. Applications. The first application of the previous results will be a multilinear version of the main theorem of [15]. We need to define first the class of Hilbert-Schmidt multilinear operators.

Definition 4.1. A multilinear operator $T: H_{1} \times \cdots \times H_{n} \rightarrow H$ between Hilbert spaces is said to be Hilbert-Schmidt if there exists $K>0$ such that

$$
\left(\sum_{i_{1} \in I_{1}, \ldots, i_{n} \in I_{n}}\left\|T\left(e_{i_{1}}^{1}, \ldots, e_{i_{n}}^{n}\right)\right\|^{2}\right)^{1 / 2}<K
$$

where $\left(e_{i_{j}}^{j}\right)_{i_{j} \in I_{j}} \subset H_{j}$ is an orthonormal basis $(1 \leq j \leq n)$. In that case, the least constant $K$ satisfying (17) is the Hilbert-Schmidt norm of $T$, which will be denoted by $\|T\|_{S_{2}}$.

The first observation we make is that the definition does not depend on the orthonormal basis chosen [11, Proposition 5.1]. It is easy to see that the class of Hilbert-Schmidt multilinear operators $S_{2}^{n}\left(H_{1}, \ldots, H_{n} ; H\right)$, with its norm $\|\cdot\|_{S_{2}}$, is a Hilbert space. The scalar product is given by

$$
(T \mid S)=\sum_{i_{1} \in I_{1}, \ldots, i_{n} \in I_{n}}\left(T\left(e_{i_{1}}^{1}, \ldots, e_{i_{n}}^{n}\right) \mid S\left(e_{i_{1}}^{1}, \ldots, e_{i_{n}}^{n}\right)\right) .
$$

ThEOREM 4.2. Let $H_{1}, \ldots, H_{n}, H$ be Hilbert spaces and $T: H_{1} \times \cdots \times$ $H_{n} \rightarrow H$ a multilinear operator. The following are equivalent:

(i) $T \in S_{2}^{n}\left(H_{1}, \ldots, H_{n} ; H\right)$.

(ii) $T \in \Pi_{p}^{n}\left(H_{1}, \ldots, H_{n} ; H\right)$ for every $p \in[1, \infty)$.

(iii) $T \in \Pi_{p}^{n}\left(H_{1}, \ldots, H_{n} ; H\right)$ for some $p \in[1, \infty)$.

Proof. (i) $\Rightarrow$ (ii) is $[11$, Proposition 5.6] and (ii) $\Rightarrow$ (iii) is trivial. To see that (iii) $\Rightarrow$ (i) we take $T \in \Pi_{p}^{n}\left(H_{1}, \ldots, H_{n} ; H\right)$. If $p \geq 2$, then [11, Proposition 5.7] tells us that $T \in S_{2}^{n}\left(H_{1}, \ldots, H_{n} ; H\right)$. If $1 \leq p \leq 2$, Theorem 3.10 yields $T \in \Pi_{2}^{n}\left(H_{1}, \ldots, H_{n} ; H\right)$. Then [11, Proposition 5.5] allows us to conclude that also in that case $T \in S_{2}^{n}\left(H_{1}, \ldots, H_{n} ; H\right)$.

Now, we are going to give the announced improvement of Theorem 1.1(ii). First, we need the following

LemMA 4.3. Let $H_{1}, \ldots, H_{n}, H$ be Hilbert spaces, $X_{1}, \ldots, X_{n}$ Banach spaces, $T: X_{1} \times \cdots \times X_{n} \rightarrow H$ a multilinear operator and $u_{j}: H_{1} \rightarrow X_{j}$ 
linear operators such that $u_{j}^{*}$ is 2 -summing for each $1 \leq j \leq n$. Then $S=$ $T\left(u_{1}, \ldots, u_{n}\right)$ is Hilbert-Schmidt and

$$
\|S\|_{S_{2}} \leq\left(\prod_{j=1}^{n} \pi_{2}\left(u_{j}^{*}\right)\right)\|T\| .
$$

Proof. The case $n=1$ is well known (see [6]). We reason by induction. If we suppose the case $n-1$ holds, we can consider the following diagram:

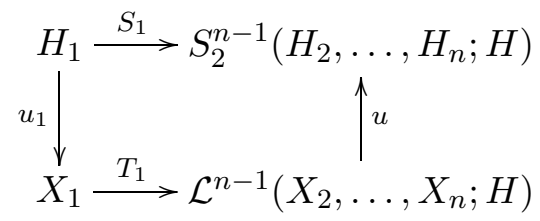

where $T_{1}, S_{1}$ are the linear operators associated to $T$ and $S$ respectively, $u(R)=R\left(u_{2}, \ldots, u_{n}\right)$ and

$$
\|u\| \leq \prod_{j=2}^{n} \pi_{2}\left(u_{j}^{*}\right) .
$$

As $S_{2}^{n-1}\left(H_{2}, \ldots, H_{n} ; H\right)$ is again a Hilbert space, the case $n=1$ shows that $S_{1}$ is Hilbert-Schmidt and

$$
\left\|S_{1}\right\|_{S_{2}} \leq\left(\prod_{j=1}^{n} \pi_{2}\left(u_{j}^{*}\right)\right)\|T\| .
$$

Now [11, Proposition 5.3] finishes the proof.

We also need the following result proved in [2].

Theorem 4.4. Let $u_{j} \in \Pi_{q}\left(X_{j}, Y_{j}\right)$ and $T \in \Pi_{p}^{n}\left(Y_{1}, \ldots, Y_{n} ; Z\right)$ and let $1 \leq r<\infty$ be such that $1 / r=1 / p+1 / q$. Then $S=T\left(u_{1}, \ldots, u_{n}\right)$ is multiple $r$-summing and $\pi_{r}(S) \leq \pi_{p}(T) \prod_{j=1}^{n} \pi_{q}\left(u_{j}\right)$.

TheOREM 4.5. Let $X_{1}, \ldots, X_{n}$ be GT spaces. Then, for any Hilbert space $H$, every multilinear operator $T: X_{1} \times \cdots \times X_{n} \rightarrow H$ is multiple 1-summing and

$$
\pi_{1}(T) \leq\left(\prod_{j=1}^{n} \mathrm{GT}\left(X_{j}\right)\right)\|T\| .
$$

Moreover, the constant $\prod_{j=1}^{n} \mathrm{GT}\left(X_{j}\right)$ is optimal.

Proof. Take finite sequences $\left(x_{i_{j}}^{j}\right)_{i_{j}=1}^{m_{j}} \subset X_{j}$ with $\left\|\left(x_{i_{j}}^{j}\right)_{i_{j}=1}^{m_{j}}\right\|_{1}^{\omega} \leq 1$ and define the operators $u_{j}: \ell_{\infty}^{m_{j}} \rightarrow X_{j}$ by $u_{j}\left(e_{i_{j}}\right)=x_{i_{j}}^{j}$. By [6, Proposition 2.2], $\left\|u_{j}\right\| \leq 1$.

By Proposition 2.2, $u_{j}$ is 2-dominated and $\Delta_{2}\left(u_{j}\right) \leq \mathrm{GT}\left(X_{j}\right)$. Therefore, for any $\varepsilon>0$, there exist Hilbert spaces $H_{j}$ and operators $v_{j}: \ell_{\infty}^{m_{j}} \rightarrow H_{j}$ 
and $w_{j}: H_{j} \rightarrow X_{j}$ such that $u_{j}=w_{j} v_{j}$ and $v_{j}, w_{j}^{*}$ are 2-summing with

$$
\pi_{2}\left(v_{j}\right) \pi_{2}\left(w_{j}^{*}\right) \leq \mathrm{GT}\left(X_{j}\right)+\varepsilon .
$$

By Lemma 4.3, $T\left(w_{1}, \ldots, w_{n}\right)$ is Hilbert-Schmidt and

$$
\left\|T\left(w_{1}, \ldots, w_{n}\right)\right\|_{S_{2}} \leq\left(\prod_{j=1}^{n} \pi_{2}\left(w_{j}^{*}\right)\right)\|T\| .
$$

Now, Theorems 1.2 and 4.4 tell us that $T\left(u_{1}, \ldots, u_{n}\right)$ is multiple 1-summing and

$$
\pi_{1}\left(T\left(u_{1}, \ldots, u_{n}\right)\right) \leq\left(\prod_{j=1}^{n}\left(\mathrm{GT}\left(X_{j}\right)+\varepsilon\right)\right)\|T\| .
$$

As $\varepsilon$ is arbitrary and $\left\|\left(e_{i_{j}}\right)_{i_{j}=1}^{m_{j}}\right\|_{1}^{\omega}=1$ in $\ell_{\infty}^{m_{j}}$, we conclude that

$$
\sum_{i_{1}, \ldots, i_{n}=1}^{m_{1}, \ldots, m_{n}}\left\|T\left(x_{i_{1}}^{1}, \ldots, x_{i_{n}}^{n}\right)\right\| \leq \pi_{1}\left(T\left(u_{1}, \ldots, u_{n}\right)\right) \leq\left(\prod_{j=1}^{n} \mathrm{GT}\left(X_{j}\right)\right)\|T\| .
$$

To see the optimality of the constant we consider, for $\varepsilon>0$, operators $u_{j}: X_{j} \rightarrow \ell_{2}$ and finite sequences $\left(x_{i_{j}}^{j}\right)_{i_{j}=1}^{m_{j}} \subset X_{j}$ such that $\left\|\left(x_{i_{j}}^{j}\right)_{i_{j}=1}^{m_{j}}\right\|_{1}^{\omega} \leq 1$ and

$$
\sum_{i_{j}=1}^{m_{j}}\left\|u_{j}\left(x_{i_{j}}^{j}\right)\right\| \geq \mathrm{GT}\left(X_{j}\right)-\varepsilon
$$

The operator $T=u_{1} \otimes \cdots \otimes u_{n}: X_{1} \times \cdots \times X_{n} \rightarrow \ell_{2}(\mathbb{N} \times \cdots \times \mathbb{N})$ has $\|T\| \leq 1$ and

$$
\sum_{i_{1}, \ldots, i_{n}=1}^{m_{1}, \ldots, m_{n}}\left\|T\left(x_{i_{1}}^{1}, \ldots, x_{i_{n}}^{n}\right)\right\|=\prod_{j=1}^{n} \sum_{i_{j}=1}^{m_{j}}\left\|u_{j}\left(x_{i_{j}}^{j}\right)\right\| \geq \prod_{j=1}^{n}\left(\mathrm{GT}\left(X_{j}\right)-\varepsilon\right) .
$$

Grothendieck's Theorem tells us that $\ell_{1}$ is a GT space with $\operatorname{GT}\left(\ell_{1}\right)=$ $K_{G}$. Thus we have the following

Corollary 4.6. Every multilinear operator $T: \ell_{1} \times \cdots \times \ell_{1} \rightarrow \ell_{2}$ is multiple 1-summing and

$$
\pi_{1}(T) \leq K_{G}^{n}\|T\|
$$

Moreover, the constant $K_{G}^{n}$ is optimal.

Finally, as another application of Theorem 3.10, we have the following general version of Theorem 1.1(ii).

Corollary 4.7. Let $X_{1}, \ldots, X_{n}$ be $G T$ spaces, $H$ a Hilbert space, $T$ : $X_{1} \times \cdots \times X_{n} \rightarrow H$ a multilinear operator and $1 \leq p \leq 2$. Then $T$ is multiple p-summing and

$$
\pi_{p}(T) \leq\left(\prod_{j=1}^{n} \sqrt{2} \mathrm{GT}\left(X_{j}\right)\right)\|T\| .
$$




\section{Open problems}

5.1. Schatten classes of multilinear operators. We have seen in Theorem 4.2 a close relation between Hilbert-Schmidt and multiple summing multilinear operators. In the linear case we have a similar relation with the Schatten spaces $S_{q}$. Specifically, we have

Theorem 5.1 ([6, Theorem 10.3]). If $2 \leq q<\infty$ and $H_{1}, H_{2}$ are Hilbert spaces, then $S_{q}\left(H_{1}, H_{2}\right) \stackrel{1}{=} \Pi_{(q, 2)}\left(H_{1}, H_{2}\right)$.

It is natural to ask if we can obtain a multilinear analogue of this result. The first question here is to find the right definition for the multilinear Schatten classes. The problem of finding this definition was first stated in [21]. By the same time, and motivated by the application of the Schatten spaces to the study of Hankel operators, this problem appeared again in [9], [13] and [14]. [3] and [4] are the first steps towards a solution.

The idea in all these papers is to define the Schatten classes $S_{q}$ of multilinear forms (they only consider the case of forms) by interpolation between the nuclear forms $S_{1}$ and the compact forms $S_{\infty}$. The first question, still open, is whether the spaces obtained by the real and complex interpolation methods coincide. What is known (see [3]) is that the definition of $S_{2}$ by interpolation coincides with Definition 4.1 for both the real and complex method.

Another possible way of defining the Schatten classes, which also appears in the above mentioned works, is to define them using approximation numbers. This alternative definition, as it appears in [14], is also closed under interpolation. However, the exact relation between these two definitions, and the relation of both of them to the class of multiple $(q, 2)$-summing operators are not clear at all.

5.2. On the Inclusion Theorem. All what is known about inclusions between the classes of multiple $p$-summing operators is contained in Theorems 3.4, 3.10 and [17, Theorem 3.6]. There are still many open questions. We point out the following:

Q1. Is it essential for $Y$ to have cotype 2 in Theorem 3.10?

Q2. Does there exist an Inclusion Theorem for $2<p \leq q<\infty$ ? If not, we could ask for weak versions of the Inclusion Theorem. Specifically, we do not know if, or under which conditions, the following is true: If $T \in \Pi_{p}^{n}\left(X_{1}, \ldots, X_{n} ; Y\right) \cap \Pi_{q}^{n}\left(X_{1}, \ldots, X_{n} ; Y\right)$ and $1 \leq p \leq r \leq$ $q<\infty$, then $T \in \Pi_{r}^{n}\left(X_{1}, \ldots, X_{n} ; Y\right)$.

Acknowledgements. The author would like to thank G. Pisier for many helpful conversations that led to a substantial improvement of this paper. The author also thanks F. Bombal and I. Villanueva for useful discussions and comments. 


\section{References}

[1] R. Alencar and M. C. Matos, Some classes of multilinear mappings between Banach spaces, Publ. Departamento de Análisis Matemático, sec. 1, no. 12, Universidad Complutense de Madrid, 1989.

[2] F. Bombal, D. Pérez-García, and I. Villanueva, Multilinear extensions of Grothendieck's theorem, to appear.

[3] F. Cobos, T. Kühn, and J. Peetre, Schatten-von Neumann classes of multilinear forms, Duke Math. J. 65 (1992), 121-156.

[4] —, - - - On $\mathfrak{S}_{p}$-classes of trilinear forms, J. London Math. Soc. 59 (1999), 10031022 .

[5] A. Defant and K. Floret, Tensor Norms and Operator Ideals, North-Holland, 1993.

[6] J. Diestel, H. Jarchow, and A. Tonge, Absolutely Summing Operators, Cambridge Univ. Press, 1995.

[7] V. Dimant, Strongly p-summing multilinear operators, J. Math. Anal. Appl. 278 (2003), 182-193.

[8] T. A. W. Dwyer III, Partial differential equations in Fischer-Fock spaces for the Hilbert-Schmidt holomorphy type, Bull. Amer. Math. Soc. 77 (1971), 725-730.

[9] S. Janson, J. Peetre, and R. Rochberg, Hankel forms and the Fock space, Rev. Mat. Iberoamericana 3 (1987), 61-138.

[10] R. Latała and K. Oleszkiewicz, On the best constant in the Khinchin-Kahane inequality, Studia Math. 109 (1994), 101-104.

[11] M. C. Matos, Fully absolutely summing and Hilbert-Schmidt multilinear mappings, Collect. Math. 54 (2003), 111-136.

[12] B. Maurey, Espaces de cotype $p, 0<p \leq 2$, in: Sém. Maurey-Schwartz 1972/73, Exp. VII, École Polyt. Palaiseau.

[13] J. Peetre, Paracommutators and minimal spaces, in: Operators and Function Theory (Lancaster, 1984), Reidel, Dordrecht, 1985, 163-224.

[14] —, Paracommutators - brief introduction, open problems, Rev. Mat. Univ. Complut. Madrid 2 (1989), 201-211.

[15] A. Pełczyński, A characterization of Hilbert-Schmidt operators, Studia Math. 28 (1967), 355-360.

[16] D. Pérez-García and I. Villanueva, A composition theorem for multiple summing operators, preprint.

[17] -, -, Multiple summing operators on Banach spaces, J. Math. Anal. Appl. 285 (2003), 86-96.

[18] —, - Multiple summing operators on $C(K)$ spaces, Ark. Mat. 42 (2004), 153-171.

[19] A. Pietsch, Absolut p-summierende Abbildungen in normierten Räumen, Studia Math. 28 (1967), 333-353.

[20] —, Operator Ideals, North-Holland, 1980.

[21] —, Ideals of multilinear functionals (designs of a theory), in: Proc. 2nd Internat. Conf. on Operator Algebras, Ideals, and their Applications in Theoretical Physics (Leipzig, 1983), Teubner, 1983, 185-199.

[22] G. Pisier, Factorization of Linear Operators and Geometry of Banach Spaces, CBMS Reg. Conf. Ser. 60, Amer. Math. Soc., 1986. 
[23] M. S. Ramanujan and E. Schock, Operator ideals and spaces of bilinear operators, Linear and Multilinear Algebra 18 (1985), 307-318.

Área Matemática Aplicada

ESCET, Universidad Rey Juan Carlos

C/ Tulipán s/n

28933 Mostoles (Madrid), Spain

E-mail: dperezg@escet.urjc.es

Received September 23, 2003

Revised version May 24, 2004 